\title{
A new optimised De Bruijn coding strategy for structured light patterns
}

\author{
Jordi Pagès, Joaquim Salvi and Josep Forest \\ University of Girona \\ Av. Lluís Santaló s/n, 17071 Girona, Spain \\ \{jpages,qsalvi,forest\}@eia.udg.es
}

\begin{abstract}
Coded structured light is an optical technique based on active stereovision that obtains the shape of objects. Oneshot techniques are based on projecting a unique light pattern with an LCD projector so that grabbing an image with a camera, a large number of correspondences can be obtained. Then, a $3 D$ reconstruction of the illuminated object can be recovered by means of triangulation. The most used strategy to encode one-shot patterns is based on De Bruijn sequences. In this paper a new way to design patterns using this type of sequences is presented. The new coding strategy minimises the number of required colours and maximises both the resolution and the accuracy.
\end{abstract}

\section{Introduction}

Coded structured light is an active stereovision technique which aims to maximise the number of correspondences that can be obtained in every image [5]. For such purpose, light patterns are projected with devices like slide or LCD projectors while a camera observes the illuminated object. The patterns are designed so that a set of points are encoded, i.e. its pattern position can be retrieved from the camera image(s) by gathering local information placed around it (decoding process). The decoded points can be triangulated obtaining a $3 \mathrm{D}$ reconstruction of the object. A key point on coded structured light techniques is to decide which coding strategy is used to generate the light patterns. The coding strategy used strongly determines the performance of the technique in terms of resolution, accuracy and number of required patterns.

This paper presents a new coded structured light technique able of obtaining shape of moving objects by projecting a unique colour encoded light pattern. The aim of the new coding strategy is to optimise the performance of the pattern in terms of resolution (number of encoded points), accuracy and robustness. The paper is structured as follows. First, in section 2 the classical De Bruijn codification for structured light patterns is introduced. Secondly, in section 3 the optimisation criteria for De Bruijn patterns are analysed. In section 4, the new coding strategy and the new pattern are presented. Then, in section 5, the algorithmic implemented steps are summarised. Afterwards, some experimental results are presented in section 6. Finally, the paper ends with the conclusions.

\section{Classical De Bruijn patterns}

The most typical pattern structures for one-shot shape acquisition are stripe-patterns (which consist of parallel adjacent bands) and multi-slit patterns (narrow bands separated by black gaps). The most effective way to encode these pattern morphologies is based on colour. The objective of any coding strategy is that, given an element of the pattern (a stripe or a slit), its position in the pattern can be obtained by inspecting a local neighbourhood of elements around it. Then, it is necessary to colour all the elements of the pattern so that every neighbourhood has its own combination of colours different to the rest. De Bruijn sequences are a mathematical resource which can achieve this aim.

A De Bruijn sequence of order $m$ over an alphabet of $n$ symbols is a string of length $n^{m}$ that contains every substring of length $m$ exactly once (known as window property). An example of binary De Bruijn sequence $(n=2)$ with window property of length $m=4$ is shown in equation 1.

$$
1000010111101001
$$

De Bruijn sequences were widely used to colour stripepatterns and multi-slit patterns by mapping every symbol of the alphabet with a certain colour [1]. The most powerful and developed technique in this field was presented by Zhang et. al [6]. The pattern proposed by Zhang et al. was a stripe-pattern composed of 125 narrow adjacent stripes coloured with 8 colours. The De Bruijn sequence used was generated with the constraint that two consecutive stripes could not have the same colour [3]. With such a constraint, the length of a De Bruijn sequence decreases from $n^{m}$ down 
to $n(n-1)^{m-1}$. The main drawback of this technique is the large number of required colours.

\section{Optimising criteria}

In the following subsections the requirements needed to optimise separately the resolution, the accuracy, the number of colours and the window size are presented.

\subsection{Maximising the pattern resolution}

Theoretically, the maximum resolution that can be reached with a unique pattern is equal to its dimension in pixels. Therefore, the ideal case would be reached when all the pixels of the pattern could be identified in the camera image. In fact, it would be only necessary to identify every row or column of the pattern, since triangulation can be done by intersecting one ray (coming from the camera) with a plane (coming from the projector). In practice, identifying every pixel-width column of the projected pattern is not feasible. The typical solution consists of downsampling the pattern resolution by joining several adjacent pixel-columns into stripes. For example, when working with LCD projectors with an horizontal resolution of 1024 pixels, projecting more than 128 stripes is not recommended.

\subsection{Maximising the accuracy}

Coded structured light techniques achieving highest accuracy project several times a periodic pattern by shifting it so that the object surface is scanned. The pattern scanned over the object surface has a square intensity profile so that a set of white slits are placed equidistantly on a black background, like in the technique proposed by Gühring [2]. The accurate detection of an intensity peak in an image is a well known problem and it can be calculated with subpixel accuracy. In stripe patterns, intensity peaks cannot be detected since the projected intensity profile is flat. Then, edges between stripes must be located. The edge position can be detected by searching for extrema in the first derivative of every one of the RGB channels. However, there exist misalignments between the extrema found in the three channels. Then, the sub-pixel position of an edge between coloured stripes cannot be calculated so accurately than an intensity peak.

\subsection{Minimising the number of colours and the window property}

The robustness of a technique based on De Bruijn coding is inversely proportional to the number of colours used and the window size. Projecting $n$ colours means that $n$ different values of the Hue space are projected in a unique pattern. The Hue of the projected colour is very sensitive to the albedo of the illuminated object and the projector and camera response function. On the other hand, the window size must be minimum in order not to violate the local smoothness assumption, which assumes that the object is smooth enough so that any encoded neighbourhood appears entire in the camera image.

\section{Towards the optimal De Bruijn pattern}

As a result of the discussions presented in the previous section, it is clear that there are several incompatibilities when the resolution, the accuracy, the number of colours and the window size must be optimised at the same time.

We propose a new kind of De Bruijn pattern which intends to optimise all the features presented in the previous section. The proposed pattern takes profit of the high resolution of the classical stripe-patterns and the accuracy of the multi-slit patterns. Concretely, we propose a pattern that in the RGB space appears as a stripe-pattern and in the Luminance or Intensity channel is a multi-slit pattern. The new coding strategy consists of defining the Intensity or Luminance component of the pattern with a square profile for every row. The difference with classical multi-slit patterns is that instead of using black gaps between the luminance peaks, we use a half-intensity level which can also contain coding information. This type of profile produces alternating maxima and minima luminance peaks in the images. In terms of RGB, the pattern has a stripe for every full-intensity region and every half-intensity region. If a De Bruijn sequence based on $n$ colours and a window property of length $m$ is mapped directly to the new pattern, the resolution obtained is $n^{m}$. However, we present a new coding strategy which achieves a resolution of $2 n^{m}$ stripes.

The new coding strategy consists of dividing the pattern into $n$ periods so that all the full-illuminated stripes inside a given period have the same colour. Then, the halfilluminated stripes of every period is coloured according to a De Bruijn sequence of order $m-1$ using the same $n$ colours used for the full-intensity stripes. Note that with this strategy two adjacent stripes can share the same colour (Hue component), but not the same luminance level. The resolution of the pattern is $2 n^{m}$ since in every period there are $n^{m-1}$ full-illuminated stripes and $n^{m-1}$ half-illuminated stripes. Furthermore, the resulting pattern has a window property of length $m$.

For our experiments, we chose a pattern with 128 stripes based on 4 colours and window property of 3 stripes encoded according to the new strategy. In figure 1, the pattern used in our experiments is shown. 
a)

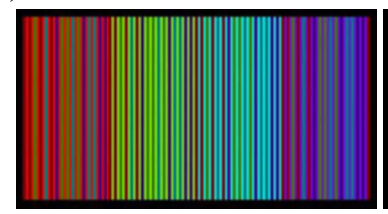

b)

Figure 1. The proposed pattern. a) RGB pattern. b) Luminance channel of the pattern

\section{Implementation and algorithmic steps}

The algorithmic implementation of the proposed coded structured light pattern is divided into two parts: the offline processing and the online processing.

\subsection{Offline processing}

The first requirement to reconstruct an object is to calibrate the projector and the camera. We have used an adaptation of the non-linear calibration algorithm proposed by Faugeras which includes a model for radial distortion [4].

Another aspect that has been calibrated is the misalignment between the three RGB channels of the camera. This misalignment is due to imperfections on the position of the CCD cells. Moreover, LCD projectors also suffer from this problem. The misalignments can reduce the accuracy of the $3 \mathrm{D}$ reconstructions. In order to minimise the global misalignment introduced by the projector and the camera, an offline procedure has been designed. Three grids are projected over a flat panel, which are coloured in red, blue and green, respectively. Then, by using the grid cross-points as reference, an homography is calculated between the red and the green channel, and another between the blue and the green channel. Applying the homographies to the images, the misalignment between the channels is minimised.

When coloured patterns are used, it must be taken into account that the perceived colours are considerably different from the projected ones. This is due to the non-linear response of both the projector and the camera, and due to the reflective properties of the object. A one-shot technique cannot estimate the albedo of the object since a unique pattern is projected, therefore, only the perturbations introduced by the projector and the camera can be minimised. The most important effect introduced by both devices is known as channel crosstalk. A robust way to minimise the crosstalk is performing a full colourimetric calibration. If a colourimeter is not available, a linear transformation between the projected and the perceived colours can be calculated. For our experiments, we chose the following RGB colours for the full-illuminated stripes: $\quad[255,0,0]^{T},[128,255,0]^{T},[0,255,255]^{T} \quad$ and $[128,0,255]^{T}$, which are equally spaced in the Hue component (the same Hue values are used for the half-illuminated stripes but with a lower luminance value). By projecting these colours over a colour-neutral panel and grabbing images, a $3 \times 3$ matrix A can be computed relating the projected colours with the observed ones.

\subsection{Online processing}

The online part of the system consists of decoding the pattern in the grabbed image and reconstructing the illuminated object by triangulating the correspondences found.

The first steps consists of removing the radial distortion of the image, reducing the RGB misalignment and rectifying the image so that horizontal scanlines can be used. Afterwards, the rows of the luminance component of the image is derived twice in order to enhance the difference between the full-illuminated stripes and the half-illuminated stripes. The derivatives are estimated by applying a linear filter of order $n$ like shown in equation 2 .

$$
g(i)=\sum_{c=1}^{n / 2}(f(i+c)-f(i-c))
$$

In our case, a filter of order 8 is sufficient. The effects of applying this filter to the luminance channel of the images is shown in figure 2. If the 2nd derivative is binarised, the regions corresponding to full-illuminated stripes and halfilluminated stripes are located. Then, in every stripe region, the sub-pixel position of the maximum or minimum peak can be accurately calculated with the Blais and Rioux peak detector as described in [2]. Peaks are searched along the rows of the image. Every located peak must be decoded in
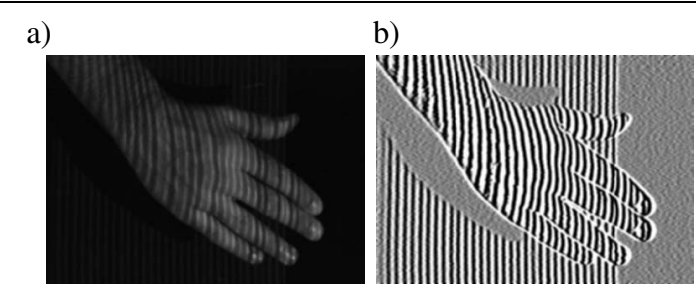

\section{Figure 2. 2nd Derivative of the luminance channel. a) Luminance image of a human hand under the pattern illumination. b) Result of applying the 2 nd derivative.}

order to know its correspondence in the projected pattern. Since the pattern has a window property of length 3 , it is necessary to identify the colours of the current stripe, and 
the two adjacent stripes. For correctly identifying the imaged colours, the inverse crosstalk matrix $\mathbf{A}^{-1}$ computed offline is used to estimate the projected colours. The last online step consists of triangulating the correspondences obtaining $3 \mathrm{D}$ points.

\section{Experimental results}

Our experimental setup consists of an XGA LCD projector Mitsubishi XL1U working at $1024 \times 768$, a camera Sony $3 \mathrm{CCD}$, a Meteor II frame grabber digitising images of $768 \times 576$ with 24-bits colour depth, and a standard PC.

A horse statue with smooth depth variation has been reconstructed as shown in figure $3 \mathrm{a}$. The proposed technique preserves the smoothness of the object and, at the same time, it detects some small details like the horse eye. The sun statue has been used for testing the resolution of the new technique. As shown in figure $3 \mathrm{~b}$, the mouth, eyes and nose of the sun are easily appreciable, and even some details of the flames. The last test consisted of reconstructing a human hand, which presented some problems. The hand texture introduces a strong gain in the red channel of the images. However, thanks to the reduced number of colours projected and the calibrated crosstalk matrix, the colours could be correctly identified and the surface was successfully reconstructed as shown in figure $3 \mathrm{c}$.
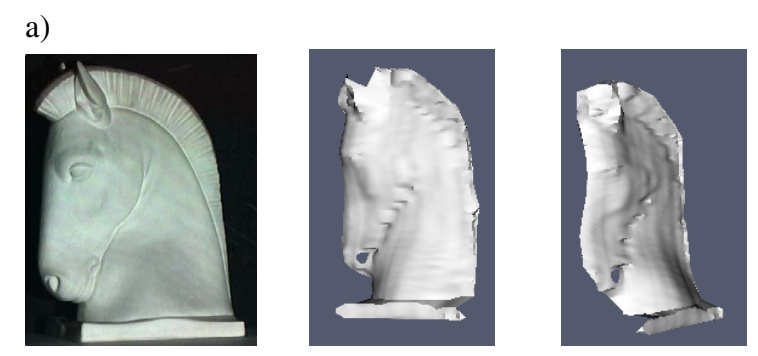

b)
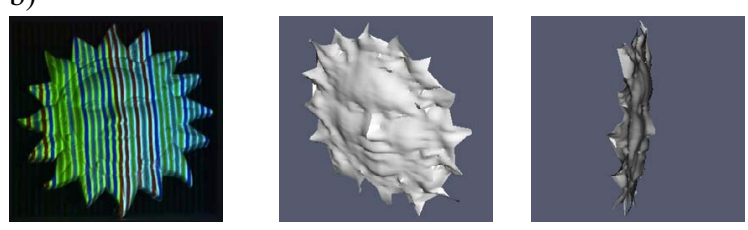

c)
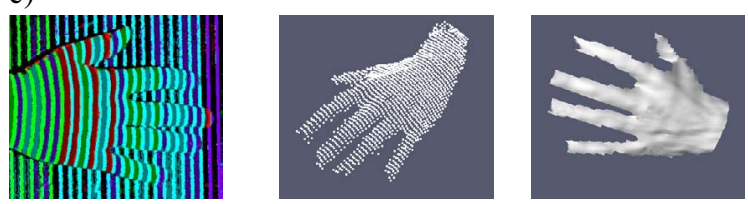

Figure 3. Reconstruction tests.

\section{Conclusions}

This paper presents a new approach to encode structured light patterns based on the well known De Bruijn sequences. The new coding strategy combines the advantages of the stripe and the multi-slit patterns in order to design new patterns where the resolution and the accuracy are maximised and the number of colours and the window size are minimised. The new pattern has a stripe appearance in the RGB space, while its Luminance component has a multi-slit structure based on two luminance levels. Therefore, coding information can be placed both in the half-illuminated stripes and in the full-illuminated stripes. Thus, we remove the constraint of the classical stripe patterns which forbid two adjacent stripes to share the same Hue value. The new coding strategy allows a pattern with $2 n^{m}$ stripes to be designed by using only $n$ colours and a window size of $m$ stripes. Remember that classical De Bruijn stripe-patterns only obtain with the same parameters $n(n-1)^{m-1}$ stripes.

A pattern consisting of 128 stripes coloured by using only 4 Hue values and a window size of 3 stripes has been implemented. Several testing objects have been reconstructed. The results demonstrate that the new pattern achieves high resolution and high accuracy taking into account that a unique image is processed.

\section{Acknowledgments}

Work funded by Spanish Project CICYT TIC200308106-CO2-02 and the Ministry of Universities, Research and Information Society, of the Catalan Government.

\section{References}

[1] C. Chen, Y. Hung, C. Chiang, and J. Wu. Range data acquisition using color structured lighting and stereo vision. Image and Vision Computing, 15:445-456, 1997.

[2] J. Gühring. Dense 3-d surface acquisition by structured light using off-the-shelf components. In Photonics West, Videometrics VII, volume 4309, pages 220-231, 2001.

[3] H. Hügli and G. Maître. Generation and use of color pseudo random sequences for coding structured light in active ranging. In Proceedings of Industrial Inspection, volume 1010, pages 75-82, Febrier 1989.

[4] J. Salvi, X. Armangué, and J. Batlle. A comparative review of camera calibrating methods with accuracy evaluation. Pattern Recognition, 35(7):1617-1635, 2002.

[5] J. Salvi, J. Pagès, and J. Batlle. Pattern codification strategies in structured light systems. Pattern Recognition, 37(4):827849, 2004.

[6] L. Zhang, B. Curless, and S. M. Seitz. Rapid shape acquisition using color structured light and multi-pass dynamic programming. In Int. Symposium on 3D Data Processing Visualization and Transmission, pages 24-36, Padova, Italy, June 2002. 